\title{
A REMARK ON THE CONSTANTS OF THE LITTLEWOOD-PALEY INEQUALITY
}

\author{
S. K. PICHORIDES
}

(Communicated by J. Marshall Ash)

Dedicated to V. Xanthopoulos

ABstract. If $f \in H^{p}, p>1$, and $\gamma$ denotes its Littlewood-Paley square function, then $\|\gamma\|_{p} \leq B_{p}\|f\|_{p}$ with $B_{p}=0\left((p-1)^{-1}\right), p \rightarrow 1^{+}$.

\section{INTRODUCTION}

Let $f$ be a $2 \pi$-periodic function in $L^{p}, 1<p<\infty, 0 \leq n_{0}<n_{1}<\cdots$ a lacunary sequence of integers, $n_{j+1} / n_{j} \geq \lambda>1, j=1,2, \ldots$, and

$$
\gamma(x)=\left\{\sum_{j=0}^{\infty}\left|S_{n_{j}}(x)-S_{n_{j-1}}(x)\right|^{2}\right\}^{1 / 2},
$$

where $S_{n}, n=0,1, \ldots$, denote the (symmetric) partial sums of the Fourier series of $f$ and $S_{n_{-1}} \equiv 0$. A well-known theorem of Littlewood and Paley asserts that

$$
A_{p}\|f\|_{p} \leq\|\gamma\|_{p} \leq B_{p}\|f\|_{p},
$$

where the positive constants $A_{p}$ and $B_{p}$ depend, for fixed $\lambda$, on $p$ only. Recently Bourgain [B] determined the asymptotic behavior of $B_{p}$ by showing

$$
B_{p}=0(p), \quad p \rightarrow \infty, \quad B_{p}=0\left((p-1)^{-3 / 2}\right), \quad p \rightarrow 1^{+} .
$$

In this note we show that this "asymmetry" disappears if we restrict ourselves to $H^{p}$ functions. More precisely we prove

Theorem. If $f \in H^{p}, p>1$, then (2) is valid with $B_{p}=0\left((p-1)^{-1}\right), p \rightarrow 1^{+}$.

It is easy to see that this estimate, as well Bourgain's estimate $B_{p}=0(p)$, $p \rightarrow \infty$, cannot be improved. To this end, take, for instance, $\lambda=2, n_{1}=N$, $n_{2}=2 N$, with $N$ sufficiently large, and consider the functions $e^{i N x} f(x)$, $e^{i N x} g(x)$, where $f, g$ are real trigonometric polynomials of degree $N$ whose conjugates $\tilde{f}, \tilde{g}$ satisfy the inequalities $\|\tilde{f}\|_{p}>C /(p-1)$ and $\|\tilde{g}\|_{p}>C p$, $1<p<\infty$. The existence of such polynomials follows easily from the estimates

Received by the editors October 15, 1990.

1980 Mathematics Subject Classification (1985 Revision). Primary 42A50, 30 D55. 
in [Z, Chapter VII, §3]. Here and in what follows the letter $C$ will denote an absolute positive constant, not necessarily the same in all its occurrences. The proof which follows has its origin in the proof of the classical theorem of Paley on $\left(H^{1}, L^{2}\right)$ multipliers [Z, Chapter XII, Theorem 7.8].

\section{PROOF OF THE THEOREM}

To facilitate the exposition we assume that $\lambda>2$. In the opposite case standard modifications of the argument lead again to the desired result at the cost of a factor which depends on $\lambda$ only. We may also assume that $f$ is a (power type) polynomial and $p<2$.

For any $g \in H^{p}$ we write $\sigma_{j}(g, x), j \geq 0$, for the (de La Vallée-Poussin type) means $\sum_{k=0}^{\infty} \hat{g}(k) \theta(k) e^{i k x}$, where the continuous function $\theta$ is 0 in $\left[n_{j+1}, \infty\right], 1$ in $\left[0, n_{j}\right]$, and linear in $\left[n_{j}, n_{j+1}\right]$. We write also $\Delta_{j}\left(\equiv \Delta_{j}(g, x)\right)$ for $\sigma_{j}-\sigma_{j-3}, j \geq 3$.

We shall make use of the following facts:

(a) $\left|\sigma_{j}(g, x)\right| \leq C M_{g}(x), j \geq 0$, where $M_{g}$ the maximal function of $g$.

(b) $f=f_{1} f_{2}$, where $f_{1}, f_{2} \in H^{2 p}$, and $\left|f_{1}\right|^{2}=\left|f_{2}\right|^{2}=|f|$.

(c) The vector valued analogue of Riesz's theorem (boundedness in $L^{p}$ of the partial sums of Fourier series) with constant $0\left((p-1)^{-1}\right), p \rightarrow 1^{+}$.

(d) The Littlewood-Paley inequality (2) in the form $\|\delta(g)\|_{p} \leq C\|g\|_{p}$ for $2<p<4$, where $\delta(g)=\left\{\sum_{j=3}^{\infty}\left|\Delta_{j}(g)\right|^{2}\right\}^{1 / 2}$ (reminder: For (a)-(c), see, e.g., [Z, Chapter IV, Theorem 7.8; Chapter VII, §7; Chapter XV, Theorem 2.15], respectively).

All these facts are well known. (d) is the basic lemma in several known proofs of (2) (see, e.g., [C, §2]).

We pass now to the proof of the theorem. Starting with the decompositions $f_{i}=\sigma_{j-3}\left(f_{i}\right)+\Delta_{j}\left(f_{i}\right)+\left(f_{i}-\sigma_{j}\left(f_{i}\right)\right), i=1,2$, and forming the product $f=f_{1} f_{2}$ we see that only the part

$$
E_{j}=\sigma_{j-3}\left(f_{1}\right) \Delta_{j}\left(f_{2}\right)+\sigma_{j-3}\left(f_{2}\right) \Delta_{j}\left(f_{1}\right), \quad j \geq 3,
$$

has spectrum which intersects $\left[n_{j-1}, n_{j}\right]$. Indeed, the part $\sigma_{j-3}\left(f_{1}\right) \sigma_{j-3}\left(f_{2}\right)$ has its spectrum in $\left[0, n_{j-1}\right)$, because of the hypothesis $\lambda>2$, and the other parts $M$ in $\left(n_{j}, \infty\right)$, because $f_{1}, f_{2} \in H^{2 p}$. On writing $\gamma_{1}=\left\{\sum_{j=3}^{\infty}\left|E_{j}\right|^{2}\right\}^{1 / 2}$ and using Riesz's theorem and (c) we obtain

$$
\begin{aligned}
\|\gamma\|_{p} & \leq \sum_{j=0}^{2}\left\|S_{n_{j}}(f)-S_{n_{j-1}}(f)\right\|_{p}+C_{p}\left\|\gamma_{1}\right\|_{p} \\
& \leq C_{p}\left(5\|f\|_{p}+\left\|\gamma_{1}\right\|_{p}\right),
\end{aligned}
$$

with $C_{p}=0\left((p-1)^{-1}\right), p \rightarrow 1^{+}$. The definition of $E_{j}$ and (a) give

$$
\gamma_{1} \leq 2 M_{f_{1}} \delta\left(f_{2}\right)+2 M_{f_{2}} \delta\left(f_{1}\right)
$$

and hence, using (d), (b), and the Hardy-Littlewood maximal function theorem [Z, Chapter I, §13],

$$
\begin{aligned}
\left\|\gamma_{1}\right\|_{p} & \leq 2\left\|M_{f_{1}}\right\|_{2 p}\left\|\delta\left(f_{2}\right)\right\|_{2 p}+2\left\|M_{f_{2}}\right\|_{2 p}\left\|\delta\left(f_{1}\right)\right\|_{2 p} \\
& \leq C\left\|f_{1}\right\|_{2 p}\left\|f_{2}\right\|_{2 p}=C\|f\|_{p}
\end{aligned}
$$


Combining (5) and (7) we obtain $\|\gamma\|_{p} \leq B_{p}\|f\|_{p}$ with $B_{p}=0\left((p-1)^{-1}\right)$, $p \rightarrow 1^{+}$, and the proof is complete.

\section{SOME REMARKS}

Remarks. (i) The above argument, for fixed $p>1$, gives $0\left((\lambda-1)^{-2}\right)$ for $B_{p}$ (the constants $C$ in (a) and (d) are replaced by $C(\lambda-1)^{-1}$ ), while examples (e.g., variants of the one used in [B] for the $(p-1)^{-3 / 2}$ result) show that it cannot be better than $0\left((\lambda-1)^{-3 / 2}\right)$.

(ii) It is not clear whether positive results for $A_{p}$ or $B_{p}, p \rightarrow \infty$, which appear to be related to good estimates for the constant in (d), can be obtained along the lines of the present proof. The example of lacunary $f$ 's and the content of [B] seem to hint toward the estimate $0\left(p^{+1 / 2}\right), p \rightarrow \infty$, for $A_{p}^{-1}$ (such an estimate, coupled with $B_{p}=0(p), p \rightarrow \infty$, leads easily to the $(p-1)^{-3 / 2}$ result). The details will appear in [P].

\section{REFERENCES}

[B] J. Bourgain, On the behavior of the constant in the Littlewood-Paley inequality, Lecture Notes in Math., no. 1376, Springer-Verlag, Berlin and New York, 1989, pp. 202-208.

[C] L. Carleson, On the Littlewood-Paley theorem, Institute Mittag-Leffler, 1967.

[P] S. Pichorides, On the Littlewood-Paley square function inequality, Colloq. Math. 60/61 (1990), 687-691.

[Z] A. Zygmund, Trigonometric series, I, II, Cambridge Univ. Press, London and New York, 1968.

Department of Mathematics, University of Crete, Iraklion, Greece

Current address: Department of Mathematics, California Institute of Technology, Pasadena, California 91125 